\title{
Are Caudal-Type Homeobox Genes Causal for Gastro-Esophageal Reflux Disease and Barrett's Esophagus?
}

\author{
Silke Laßmann • Martin Werner
}

Published online: 20 September 2013

(c) Springer Science+Business Media New York 2013

In this issue of Digestive Diseases and Sciences, Ren et al. [1] report on "Single nucleotide polymorphisms of Caudal type homeobox 1 and 2 are associated with Barrett's esophagus", the latter considered a precursor lesion of esophageal cancers.

With this, the authors address two esophageal diseases which are of rising incidence in the Western world and which are considered as precursor lesions of esophageal cancers [2]: gastroesophageal reflux disease (GERD) and Barrett's esophagus (BE). BE is characterized by transdifferentiation of the normal squamous epithelial cells to columnar/intestinal type cells of the esophageal lining. The associated patient management implications, such as screening of patients with GERD or BE are complex and frequently discussed.

Thus it is increasingly evident that the identification and validation of risk factors for GERD and especially $\mathrm{BE}$ are an important prerequisite for future decisions regarding patient management, also in view of potential preventive measurements for esophageal cancers.

Accepted risk factors which contribute to development of GERD are age, gender and abdominal obesity, which together with GERD the presence of a hiatal hernias and tobacco use are known risk factors for BE development [2-4]. Further risk stratification of $\mathrm{BE}$ patients was addressed by

S. Laßmann · M. Werner $(\bowtie)$

Department of Pathology, University Medical Center, Freiburg,

Germany

e-mail: direktion-pathologie@uniklinik-freiburg.de

S. Laßmann

e-mail: silke.lassmann@uniklinik-freiburg.de

S. Laßmann $\cdot$ M. Werner

German Cancer Consortium (DKTK) and German Cancer

Research Center (DKFZ), Heidelberg, Germany clinical-pathological and molecular studies, but so far the presence of dysplasia in the metaplastic epithelium has been established as the only validated risk factor for BE progression to esophageal cancer [5]. Also, the progression from $\mathrm{BE}$ to dysplasia and carcinoma is rare $(0.12-0.5 \%)[5,6]$.

In patients with GERD, bile and gastric acids reach the esophageal epithelial lining. The acid damaged epithelium may react by trans-differentiation from squamous to columnar/intestinal type of cells in order to counteract the bile acid damage by mucin producing cells. These consequences of long-standing GERD are accompanied by endoscopically visualized erosive or ulcerative lesions in about $1 / 3$ of patients. The diagnosis of $\mathrm{BE}$ is therefore based on histologic examination of biopsies in addition to endoscopic findings. Although several environmental and molecular factors were linked to either GERD or BE, their causative role as risk factors for the progression of GERD to BE or even for the direct development of BE remain unclear.

Ren et al. [1] in this issue of DDS used a hypothesisdriven approach to answer this question. In their study, the interdisciplinary team of researchers in the fields of cancer research, biotechnology, mathematics and gastroenterology addressed whether or not the presence of ten pre-selected single nucleotide polymorphisms (SNPs, i.e. a DNA sequence variation present in $>1 \%$ of the population) in two genes controlling columnar/intestinal differentiation, namely, caudal-type homeobox 1 and 2 (CDX1 and CDX2), confer a higher risk for the development of BE. Moreover, they also investigated two genomic loci that predispose to $\mathrm{BE}$ development recently identified in a large genome-wide association study [7].

For their analyses, the authors studied blood samples from 109 patients with BE and compared these to samples from 223 patients with either erosive esophagitis (EE) or endoscopically-negative reflux disease (ERD) (both 
grouped together and termed GERD). By allelic discrimination assay of the ten pre-selected SNPs, the authors reported that 3/5 CDX1 and 2/5 CDX2 SNPs as well as one CDX1 haplotype were statistically correlated with $\mathrm{BE}$, but not with GERD (EE + ERD). The two previously reported BE-associated genomic loci [7] were however not significantly associated with BE. This apparent discrepancy may have been due to differing study designs: the study by Ren et al. [1] was based on samples from patients with EE, ERD or BE, whereas the study by Su et al. [7] examined samples of histologically confirmed BEs and healthy controls (more than 1,000 in each group).

The study by Ren et al. [1] hence focuses on a highly relevant scientific question, the clarification of which may eventually improve stratification or preventive measurements of patients at risk for BE, providing some key insights and scientific basis for further investigation.

First, in this retrospective study design, patients with endoscopically and histologically diagnosed $\mathrm{BE}$ were compared with patients with EE or with ERD. Only single samples were obtained from each patient with no follow-up or available prospective samples, especially from those with GERD. Thus it remains unclear whether or not EE and ERD patients with specific SNP profiles would have later developed $\mathrm{BE}$. Indeed, since $\mathrm{BE}$ is considered to be a complication of GERD [2-4], it is logical to assume that similar SNP profiles are present in GERD and BE patients, if these SNPs are causative risk factors. Therefore, the data by Ren et al. [1] show a clear association of selected SNPs with BE, although association does not prove causation. Proving causation for selected SNPs is further complicated by the observation that the selected and BE-correlated SNPs were themselves all significantly associated with BE risk factors such as age, sex and hiatal hernias [2, 3]. Indeed, a recent study established a clinical risk model for progression of GERD to $\mathrm{BE}$, based on age, gender, smoking habits, weight, use of acid suppressants and, quite surprisingly also education [4].

A clear distinction between risk, susceptibility, and association of molecular findings with esophageal disease and potential cancer predisposition is hence of importance. Ren et al. [1] promise to identify if the SNPs are indeed causative risk factors, useful for risk stratification. Whether or not such molecular screening represents a major priority for care of esophageal diseases is however still debatable, since the risk for development of BE and further dysplasia or carcinoma is fortunately low $[5,6]$.

The second most interesting aspect in the study by Ren et al. [1] is the selection of SNPs affecting the two caudal type homeobox genes CDX1 and CDX2. Clearly, the histologic appearance of $\mathrm{BE}$ is associated with specialized trans-differentiated columnar/intestinal type of cells, which presumably evolve via genetic or epigenetic mechanisms.
So far, CDX2, but not CDX1 is a useful marker for the routine diagnosis of $\mathrm{BE}$, although also CDX1 is clearly linked with BE [8]. Causes for altered gene expression of CDX1 or CDX2 may be manifold, including nitric oxideinduced altered epithelial growth factor receptor (EGFR) or nuclear factor kappa-light-chain-enhancer of activated $\mathrm{B}$ cells (NFKB) signaling, as also mentioned by Ren et al. [1]. In the present study, no biopsies were studied for epigenetic regulation (e.g. methylation) and expression of CDX1 or CDX2. SNPs may affect transcriptional profiles or protein expression and thereby define phenotype (such as GERD or BE). Therefore further analyses of CDX proteins in patient-matched biopsies of EE, ERD or BE may give a hint towards whether or not the characteristic CDX expression in BE is driven-at least in part-by the particular SNPs. Moreover, other genes are associated with development of BE, such as the MUC gene family and liver-intestine cadherin $[9,10]$. Therefore, future prospective studies as envisaged by Ren et al. may benefit from the inclusion of additional SNPs in genes presumably guiding the columnar to intestinal trans-differentiation.

In summary, GERD and BE are multifactorial genetic and epigenetic diseases that may be caused by specific SNPs in key regulatory genes. For SNPs to be considered as disease-causing rather than simply as biomarkers requires large-scale studies and additional information about their functional consequences. The approach by Ren et al. [1] is a major step towards reaching this aim by providing novel starting points for further investigation.

\section{References}

1. Ren D, Zheng G, Bream S, Tevebaugh W, Shaheen NJ, Chen X. Single nucleotide polymorphisms of caudal type homeobox 1 and 2 are associated with Barrett's esophagus. Dig Dis Sci. [Epub ahead of print]. doi:10.1007/s10620-013-2804-9.

2. Bosman FT, Carneiro F, Hruban RH, Theise ND. WHO classification of tumours of the digestive system. In: WHO classification of tumours, vol 3, 4th edn. IARC, Lyon, France

3. Pohl H, Wrobel K, Bojarski C, et al. Risk factors in the development of esophageal adenocarcinoma. Am J Gastroenterol. 2013;108:200-207.

4. Thrift AP, Kendall BJ. A clinical risk prediction model for Barrett esophagus. Cancer Prev Res (Phila). 2012;5:1115-1123.

5. Hvid-Jensen F, Pedersen L, Drewes AM, et al. Incidence of adenocarcinoma among patients with Barrett's esophagus. $N$ Engl J Med. 2011;365:1375-1383.

6. Lenglinger J, Riegler M, Cosentini E, et al. Review on the annual cancer risk of Barrett's esophagus in persons with symptoms of gastroesophageal reflux disease. Anticancer Res. 2012;32: $5465-5473$.

7. Su Z, Gay LJ, Strange A, for the Esophageal Adenocarcinoma Genetics Consortium, et al. Welcome Trust Case Control Consortium 2. Common variants at the MHC locus and at chromosome 16q24.1 predispose to Barrett's esophagus. Nat Genet. 2012;44:1131-1136. 
8. Wong NA, Wilding J, Bartlett S, et al. CDX1 is an important molecular mediator of Barrett's metaplasia. Proc Natl Acad Sci USA. 2005;102:7565-75670.

9. Weimann A, Zimmermann M, Gross M, et al. CDX2 and LIcadherin expression in esophageal mucosa: use of both markers can facilitate the histologic diagnosis of Barrett's esophagus and carcinoma. Int J Surg Pathol. 2010;18:330-337.
10. Khor TS, Alfaro EE, Ooi EM, et al. Divergent expression of MUC5AC, MUC6, MUC2, CD10, and CDX-2 in dysplasia and intramucosal adenocarcinomas with intestinal and foveolar morphology: is this evidence of distinct gastric and intestinal pathways to carcinogenesis in Barrett Esophagus? Am J Surg Pathol. 2012;36:331-342. 